\title{
BACIA HIDROGRÁFICA DO RIO MEQUÉNS: LEVANTAMENTO ETNOZOOLÓGICO DA ICTIOFAUNA EM UMA COMUNIDADE TRADICIONAL RIBEIRINHA
}

\author{
HYDROGRAPHIC BASIN OF THE MEQUÉNS RIVER: ETHNOZOOLOGICAL SURVEY \\ OF THE ICHTHYOFAUNA IN A TRADITIONAL RIVERSIDE COMMUNITY
}

\author{
Bruno Elias Rocha LOPES ${ }^{*}$, Fernanda Cristina Gonsalves THOMAZIN², Mário Gorza ROMANO³ \\ ${ }^{1}$ Mestre em Ensino de Física, Docente, Departamento de Farmácia, Faculdade São Paulo - FSP, Av. 25 de Agosto, 6961 - São Cristóvão, Rolim de Moura - RO, \\ 76940-000, *brunoerl@gmail.com \\ ${ }^{2}$ Estudante, Escola Estadual de Ensino Médio em Tempo Integral Candido Portinari, Av. Fortaleza, 5550 - Centro, Rolim de Moura - RO, 76940-000, \\ fernandathomazin@gmail.com \\ ${ }^{3}$ Especialista em Docência do Ensino Superior, Departamento de Engenharia, Faculdade São Paulo - FSP, Av. 25 de Agosto, 6961 - São Cristóvão, Rolim de \\ Moura - RO, 76940-000, mariogzromano@gmail.com
}

\begin{abstract}
RESUMO: O presente estudo desenvolveu-se no distrito de Rolim de Moura do Guaporé - RO, localizado em uma ilha no Rio Mequéns, afluente da margem direita do Rio Guaporé, formado por uma comunidade de diversidade étnica localizada próxima a uma reserva ambiental e cercada por floresta nativa. O objetivo consistiu no levantamento etnozoológico de peixes, partindo do conhecimento dos moradores locais. Metodologicamente, consistiu no levantamento qualitativo do número de espécies presentes naquele espaço geográfico, através de um formulário semiestruturado abordando nomes populares dos animais e períodos do dia nos quais eram avistados. Vale ressaltar que também foram exibidas imagens dos animais citados para a confirmação das espécies e período de atividade. Os formulários foram desenvolvidos e aplicados pelos alunos da EEEMTI Cândido Portinari - Rolim de Moura - RO, sob a orientação dos professores de Biologia, Física e Geografia. Foram entrevistadas 23 pessoas, sendo: 12 do sexo masculino e 11 do feminino, com idade entre $12 \mathrm{e}$ 87 anos, com tempo de habitação no local estabelecido num período entre 04 a 87 anos. Como resultado, obteve-se uma lista na qual os animais mais citados foram: pintado (Pseudoplatystoma corruscans), tucunaré (Cichla sp.), pirarara (Phractocephalus hemioliopterus) e piau (Leporinus sp.). A maioria dos avistamentos ocorreu durante o período diurno (manhã e tarde). Todos os fatos relatados aconteceram enquanto os entrevistados estavam pescando, por lazer ou como fonte de renda. A pesquisa mostrou que a população local possui riqueza de informações que facilita inventariar a fauna da região.
\end{abstract}

Palavras-chave: Etnobiologia, biodiversidade, Peixes, Rondônia, Meio ambiente.

ABSTRACT: The present study was carried out in the district of Rolim de Moura do Guapore - RO, located on an island in the Mequéns River affluent of the right bank of the Guaporé River, formed by a community of ethnic diversity located next to an environmental reserve and surrounded by native forest. The objective consisted of an ethno-zoological survey of fish, based on the knowledge of local residents. Methodologically, it consisted of a qualitative survey of the number of species present in that geographic space, through a semi-structured questionnaire addressing: popular names of animals and periods of the day in which they were sighted. It is worth mentioning that images of the cited animals were also displayed to confirm the species and activity period. The questionnaires were developed and applied by students of EEEMTI Cândido Portinari - Rolim de Moura - RO, under the guidance of Biology, Physics and Geography teachers. 23 people were interviewed, being: 12 male and 11, female, aged between 12 and 87 years, with time of residence in the place established in a period between 04 to 87 years. As a result, a list was obtained in which the most cited animals were: painted (Pseudoplatystoma corruscans), tucunaré (Cichla sp.), pirarara (Phractocephalus hemioliopterus) and piau (Leporinus sp.). Most sightings occurred during the daytime (morning and afternoon). All the facts reported happened while the interviewees were fishing, for leisure or as a source of income. The research showed that the local population has a wealth of information that makes it easy to inventory the fauna of the region.

Keywords: Ethnobiology, biodiversity, Fish, Rondônia, Environment 


\section{INTRODUÇÃO}

O Brasil é considerado o país com maior diversidade de peixes dulcícolas, com a estimativa de 2.587 espécies. Nesse cenário, destaca-se a Bacia Amazônica que abriga cerca de $20 \%$ da água doce presente no mundo (Rezende et al., 2008; Lewinsohn e Prado, 2005), possuindo uma área de 6,5 milhões de $\mathrm{km}^{2}$ (Goulding, 1996), podendo chegar a 7,1 milhões de $\mathrm{km}^{2}$ considerando a bacia dos Rios Tocantins/Araguaia (Junk, 1980), sendo possuidora de grande biodiversidade de animais aquáticos, principalmente ictiofauna, que se revelam vitais para a manutenção de todo esse ecossistema aquático (Oliveira, 2014), podendo chegar a um total 8.000 espécies (Schaefer,1998), sendo elas de água doce neotropicais, representando um oitavo de toda biodiversidade estimada de vertebrados viventes (Vari e Malabarba, 1998).

Nesse contexto, a sub-bacia do rio Guaporé possui aproximadamente 700 mil hectares, localizada entre $\mathrm{S} 12^{\circ} 35^{\prime}$ e $13^{\circ} 30^{\prime}$ e $\mathrm{O} 60^{\circ} 55^{\prime}$ e $64^{\circ} 20$. Ao sul dessa região, encontra-se o Chaco boliviano, o qual detém campos inundáveis e formações aluviais, entremeados por grandes lagos. Por outro lado, ao norte, está situada a floresta amazônica de terra firme (floresta ombrófila) e a leste, uma faixa de transição entre os biomas da Amazônia e do Cerrado (Fernandes e Guimarães, 2002)

Igualmente, o rio Guaporé encontra-se localizado nos Estados de Mato Grosso e Rondônia. Assim, em grande parte de seu trajeto, o Guaporé estabelece uma fronteira natural entre Brasil e Bolívia. Possui sua nascente na Fazenda São Paulo, na Serra dos Parecis - MT, a uma altitude de 630 metros e sua Foz no Rio Mamoré, a 131 metros de altitude. Sua extensão equivale a aproximadamente 1.470 km. Ademais, por constar na bacia Amazônica, localiza-se nos Estados de Mato Grosso e Rondônia, passando pelos biomas do Cerrado, Pantanal e Floresta Amazônica. No total, 11 são os municípios contíguos ao rio, nas margens brasileiras, sendo: Vale de São Domingos, Pontes e Lacerda, Vila Bela da Santíssima Trindade, Comodoro, Cabixi, Pimenteiras do Oeste, Alto Alegre dos Parecis, Alta Floresta d'Oeste, São Francisco do Guaporé, Costa Marques e Guajará-Mirim, os quais totalizam uma população de 206.111 habitantes (Moss e Moss, 2007).

Importante ressaltar que suas águas possuem cor negra, ausentes de poluição e com margens pouco perturbadas. Nas margens desse rio, encontram-se vários lagos, que são conhecidos como "baías" pela população local, que se conectam com o rio durante a cheia. Ao longo da época da vazante, que dura entre setembro e novembro, ocorre o isolamento de algumas baías e outras mantêm a comunicação com o rio Guaporé (Fachín-Terán e Vogt, 2004), sendo que, ao rio Méquens, une-se dentro do Parque Estadual Corumbiara, no Estado de Rondônia (Nunes, 2016). Quanto a sua ictiofauna, possui predominantemente as espécies das ordens Characiformes e Siluriformes (de Oliveira, 2014).

Uma das características mais conhecidas de comunidades presentes na Amazônia é o seu conhecimento tradicional, construído ao longo de várias gerações, tanto sobre região ou ambiente em que vivem, quanto sobre diversidade das espécies de animais e vegetais presentes naquele ecossistema. Tal cognição foi sendo construída devido à necessidade de se ter a floresta como meio de sobrevivência e forma de recurso financeiro. No caso de ambientes aquáticos, a relação diária e as necessidades do ribeirinho permitiram que, através de suas experiências, adquirissem a habilidade de reconhecer, com ciência, a distribuição das espécies exploradas, os movimentos migratórios e as variações sazonais (Doria et al., 2008).

Este trabalho objetivou realizar um levantamento etnozoológico dos peixes que são pescados na região de Rolim de Moura do Guaporé - RO, a partir de entrevistas realizadas com moradores locais. $\mathrm{O}$ presente estudo faz-se necessário, tendo em vista existir carência desse tipo de estudo e de informações sobre a ictiofauna da região. Além disso, o etnoconhecimento sobre a fauna tem sido uma ferramenta pouco explorada em pesquisas científicas, mesmo tendo em vista que as comunidades tradicionais locais são possuidoras de informações relevantes para tal finalidade, tornando seu uso um instrumento importante para a pesquisa.

\section{MATERIAL E MÉTODOS}

Nessa temática, o presente estudo consiste no levantamento qualitativo do número de espécies de peixes que são avistados pela população de Rolim de Moura do Guaporé - RO, presente nas imediações do Rio Guaporé. Com essa finalidade, foi elaborado um formulário semiestruturado o qual abordou: i) quais animais são avistados com maior frequência (sendo que cada entrevistado poderia citar até três espécies); e ii) períodos do dia (manhã, tarde e/ou noite) em que foram avistados (podendo ser relatado mais de um período para cada espécie, caso houvesse o encontro). Em seguida, foram exibidas imagens dos animais citados para a confirmação das espécies e período de atividade. 
Ademais, os formulários foram desenvolvidos e aplicados pelos estudantes da E.E.E.MTI. Cândido Portinari - Rolim de Moura - RO, sob a orientação dos professores de Biologia, Física e Geografia. Como resultado, auferiu-se uma listagem, a qual serviu de instrumental para o presente trabalho.

Importante destacar que a presente pesquisa foi desenvolvida nos dias 23 e 24 de novembro de 2017, dividida em dois períodos de coleta de dados, sendo das 8:00 às 12:00 e das 15:00 às 17:00. Foram entrevistados indivíduos através de encontros aleatórios na rua, em estabelecimentos comerciais, casas e órgãos públicos. Os estudantes tomaram o cuidado de evitar passar pelo mesmo logradouro mais de uma vez.

\section{Área de estudo}

O presente estudo desenvolveu-se no distrito de Rolim de Moura do Guaporé - RO (Figuras 1 e 2), localizado no afluente da margem direita do Rio Guaporé, às margens do Rio Mequéns, o qual é formado por uma comunidade ribeirinha com diversas etnias, entre as quais indígenas e descendentes de quilombolas. O distrito está localizado próximo à reserva ambiental do Guaporé, sendo essa comunidade cercada por floresta nativa, sendo muito requisitada para práticas recreativas, pesca esportiva e contato com a natureza (Stachiw, 2017). Situa-se na região do Pantanal rondoniense, fazendo fronteira com a Bolívia, na área territorial de 2.225,170 $\mathrm{km}^{2}$. Localiza-se às margens do rio Mequéns, afluente do Guaporé, no entorno do Parque Estadual de Corumbiara, local onde o acesso só é possível por vias fluviais ou aéreas (Nunes, 2016).

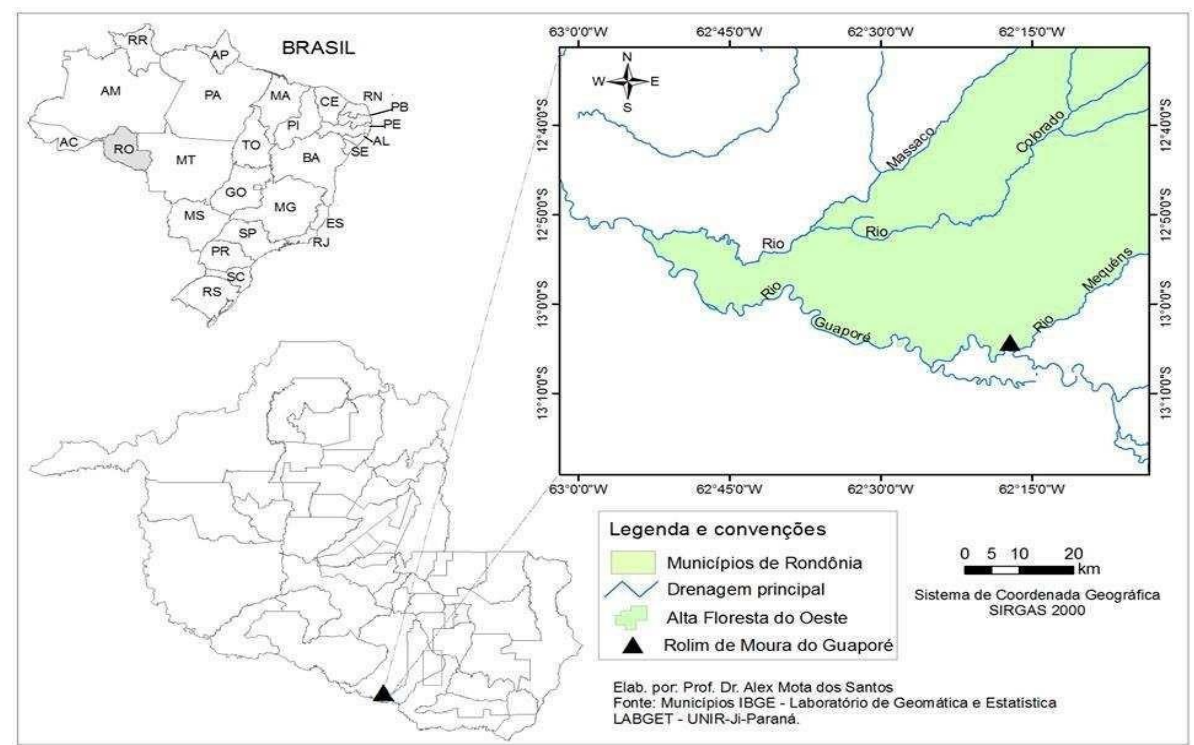

Figura1: Localização do Distrito de Rolim de Moura do Guaporé, Rondônia. (Fonte: NUNES, 2016).

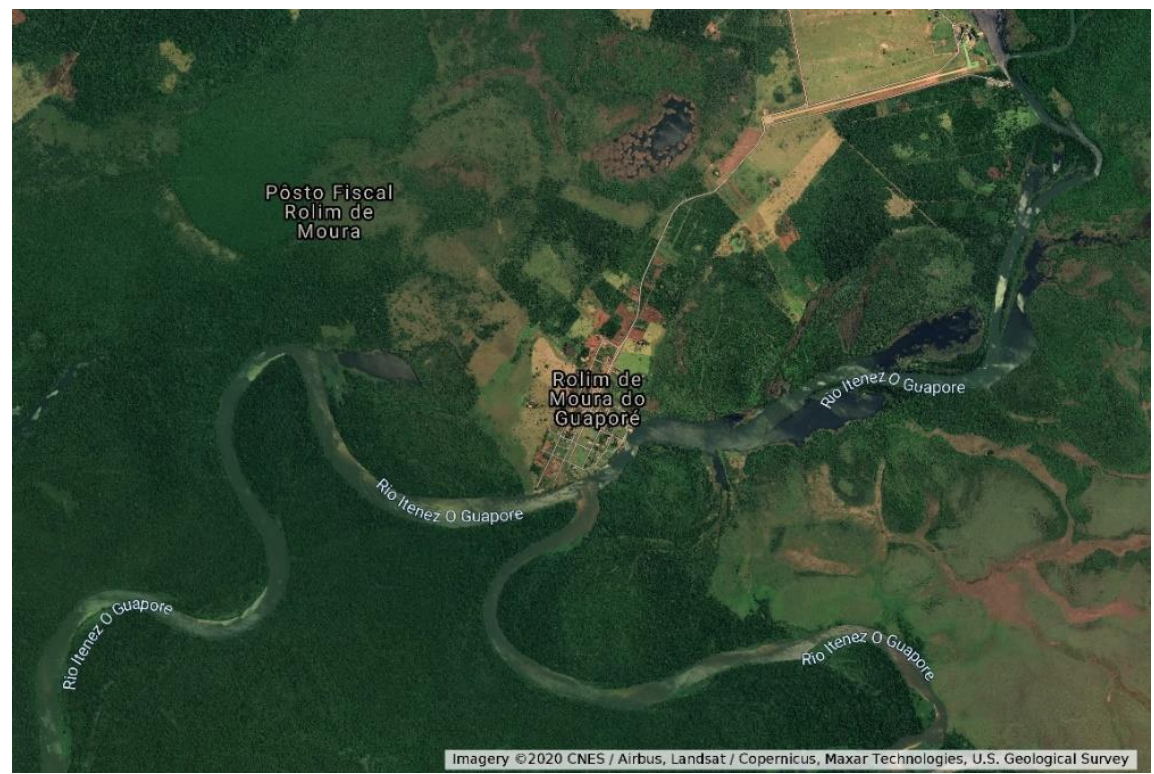

Figura 2: Imagem de satélite do distrito de Rolim de Moura do Guaporé (Fonte: Google Earth Pro, 2020). 
O distrito pertence ao município de Alta Floresta D’Oeste - RO. Possui uma população de 25.437 pessoas, sendo sua área territorial de $7.067,025 \mathrm{~km}^{2}$ (IBGE, 2017). O clima predominante é o tropical quente úmido com temperatura média de $26^{\circ} \mathrm{C}$, apresentando períodos de seca e de chuva. Além disso, os meses mais chuvosos ocorrem entre novembro a abril com, aproximadamente $90 \%$ da precipitação pluviométrica verificada nesse intervalo temporal (Oliveira, 2002).

\section{RESULTADOS E DISCUSSÃO}

Participaram das entrevistas 23 pessoas, sendo 12 do sexo masculino e 11 do feminino. A faixa etária oscilando entre 12 e 87 anos, bem como com tempo de habitação no local variando entre 04 a 87 anos. Foram relatados, no total, 11 nomes populares que resultaram em 12 espécies diferentes (Quadro 1), dos quais os mais citados foram: pintado (Pseudoplatystoma corruscans), tucunaré (Cichla sp.), pirarara (Phractocephalus hemioliopterus) e piau (Leporinus sp.).

Quadro 1 - Relação qualitativa e quantitativa das citações.

\begin{tabular}{|l|c|}
\hline Nomes popular e científico dos peixes & Quantidade de citações \\
\hline Pintado (Pseudoplatystoma corruscans) & 13 \\
\hline Tucunaré (Cichla sp.) & 10 \\
\hline Pirarara (Phractocephalus hemioliopterus) & 5 \\
\hline Piau (Leporinus sp.) & 4 \\
\hline Piranha (Pygocentrus nattereri / Serrasalmus sp.) & 4 \\
\hline Traíra (Hoplias lacerdae) & 3 \\
\hline Matrinxa (Brycon cephalus) & 3 \\
\hline Tambaqui (Colossoma macropomum) & 2 \\
\hline Cachara (Pseudoplaystoma fasciatum) & 1 \\
\hline Curimba (Prochilodus sp.) & 1 \\
\hline
\end{tabular}

A presença do pintado (Pseudoplatystoma corruscans) condiz com o trabalho de Arruda (2018), quem cita a presença dessa espécie nas comunidades quilombolas Retiro, Boqueirão, Casalvasco e Manga, localizadas na zona rural do município de Vila Bela da Santíssima Trindade, no Alto Guaporé - MT.

A presença de Tucunaré (Cichla sp.) concorda com trabalho de Oliveira (2014), o qual relatou a presença dessa espécie no Rio Guaporé, na região de Pontes e Lacerta - MT, assim como Doria et al. (2008), que também explanaram sobre a presença de pirarara (Phractocephalus hemioliopterus), curimba (Prochilodus sp.), piranha (Serrasalmus rhombeus) e tambaqui (Colossoma macropomum) na região da colônia de pescadores de Costa Marques - RO.

Em relação ao período do dia (Quadro 2), a maioria dos encontros ocorreu durante o período diurno, os quais totalizaram 51 avistamentos, ocorrendo a maioria deles no período matutino (28, no total). O peixe com maior número de encontros apontados foi o pintado (15), sendo 13 no período diurno (manhã e tarde), com predominância de 10 avistamentos apenas durante o período vespertino. Seguido pelo tucunaré, que foi avistado 12 vezes (11, no período diurno), sendo a manhã o período com maior atividade. Por outro lado, piranha e piau totalizaram sete relatos cada, somando todos os períodos apresentados no formulário. Destaca-se que, durante a manhã, o peixe com maior número de relatos foi o tucunaré. Na sequência, no horário vespertino, pintado, ao passo que no noturno, piau.

Quadro 2 - Relação de número de avistamentos de espécies de peixes por período.

\begin{tabular}{|l|c|c|c|}
\hline \multirow{2}{*}{ Animal citado - Nome Popular e Cientifico Peixes } & Quantidade de Citações & Noite \\
\cline { 2 - 4 } & Manhã & Tarde & 2 \\
\hline Pintado (Pseudoplatystoma corruscans) & 3 & 10 & 1 \\
\hline Tucunaré (Cichla sp.) & 7 & 4 & 1 \\
\hline Pirarara (Phractocephalus hemioliopterus) & 3 & 2 & 3 \\
\hline Piau (Leporinus sp.) & 2 & 2 & 2 \\
\hline Piranha (Pygocentrus nattereri / Serrasalmus sp.) & 4 & 1 & - \\
\hline Traíra (Hoplias lacerdae) & 3 & 2 & - \\
\hline Matrinxa (Brycon cephalus) & 3 & - & - \\
\hline Tambaqui (Colossoma macropomum) & 2 & 1 & 1 \\
\hline Cachara (Pseudoplaystoma fasciatum) & 1 & - & - \\
\hline Curimba (Prochilodus sp.) & - & 1 & \\
\hline
\end{tabular}




\section{CONCLUSÃO}

Em síntese, a pesquisa mostrou que o local possui uma ictiofauna variada, quando relacionada ao contato com o ser humano, sendo a mesma mais avistada ao longo do dia do que da noite, o que pode coincidir com o período de maior atividade humana no rio. Também foi notado que o conhecimento tradicional pode ser uma ferramenta útil para o inventariamento da ictiofauna de uma região, pois o contato próximo que a comunidade ribeirinha mantém com o rio, devido à atividade pesqueira, bem como os recursos naturais disponíveis, permite a aquisição maior de conhecimentos sobre a fauna regional de peixes.

\section{REFERÊNCIAS}

ARRUDA, J. C. et al. Traditional ecological knowledge of fish fauna in quilombolas on the Alto Guaporé, Mato Grosso, Southern Amazonia, Brazil. Boletim do Museu Paraense Emílio Goeldi. Ciências Humanas, v. 13, n. 2, p. 315-329, 2018.

DORIA, C. R. et al. Contribuição da etnoictiologia à análise da legislação pesqueira referente ao defeso de espécies de peixes de interesse comercial no oeste da Amazônia Brasileira, rio Guaporé, Rondônia, Brazil. Biotemas, v. 21, n. 2, p. 119-132, 2008.

DE OLIVEIRA, M. A. et al. Ictiofauna do Rio Guaporé e áreas adjacentes. PUBVET, v. 8, p. 0975-1135, 2014.

FACHÍN-TERÁN, A.; VOGT, R.C. Estrutura populacional, tamanho e razão sexual de Podocnemis unifilis (Testudines, Podocnemididae) no rio Guaporé (RO), norte do Brasil. Phyllomedusa, v. 3, n. 1, p. 29-42, 2004.

FERNANDES, L. C.; GUIMARÃES, S. C. P. (orgs). Atlas Geoambiental de Rondônia. Porto Velho: SEDAM/GOVERNO DE RONDÔNIA, 2002.

GOULDING, M. Pescarias amazônicas, proteção de habitats e fazendas nas várzeas: uma visão ecológica e econômica. Relatório técnico para o projeto (Manejo dos recursos naturais da Várzea). Brasília: IBAMA, 1996.

IBGE (Instituto Brasileiro de Geografia e Estatística). Brasil em Síntese - Rondônia - Alta Floresta D'Oeste. Disponível em: <https://cidades.ibge.gov.br/brasil/ro/alta-floresta-doeste/panorama〉. Acessado em: 10 ago 18

JUNK, W. J. Áreas inundáveis: um desafio para a limnologia. Acta Amazonica, v. 10, n. 4, p. 775-796, 1980.

LEWINSOHN, T. M.; PRADO, P. I. Quantas espécies há no Brasil? Megadiversidade, v. 1, p. 36-42, 2005.

OLIVEIRA, O. A. Geografia de Rondônia: espaço e produção. Dinâmica, Porto Velho, 2002.

MOSS, G.; MOSS, M. Projeto Brasil das águas: Sete Rios, Guaporé. Brasília, 2007. Disponível em: <http://brasildasaguas.com.br/wp-content/uploads/sites/4/2013/05/Rio-Guapore\%C2\%81_Relatorio.pdf $>$. Acesso em: 25 ago 2020.

NUNES, R. O. Prospecção etnofarmacológica de plantas medicinais utilizadas pela população remanescente de quilombolas de Rolim de Moura do Guaporé, Rondônia, Brasil. 2016. 200 f. Tese (Doutorado em Fitotecnia) - Universidade Federal de Viçosa, Viçosa. 2016.

REZENDE, F. J. W. et al. Perfil da aquicultura no estado do Acre. Amazônia: Ciência e Desenvolvimento, v. 4, n. 7, 2008

SCHAEFER, S. A. Conflict and resolution: impact of new taxa on phylogenetic studies of the Neotropical cascudinhos.In: Malabarba, R. L. et al. (eds). Phylogeny and classification of Neotropical Fishes. Porto Alegre: Edipucrs, 1998. p. 375-400.

STACHIW, R. Cartilha das águas de Rondônia. Rolim de Moura: Universidade Federal de Rondônia, 2017.

VARI, R.P.; MALABARBA, L.R. Neotropical ichthyology. In: MALABARBA, L. R. et al. (eds). Phylogeny and classification of Neotropical fishes. Porto Alegre: Ed. PUCRS, 1998. p. 1-11. 